\title{
Characteristics of sexually experienced HIV testers aged 18 to 32 in rural South Africa: baseline results from a community-based trial, NIMH Project Accept (HPTN 043)
}

Lucia Knight ${ }^{*}$, Nuala McGrath ${ }^{2,3}$, Heidi van Rooyen ${ }^{4}$, Hilton Humphries ${ }^{4}$, Alastair van Heerden ${ }^{4,5}$ and Linda Richter $5,6,7$

\begin{abstract}
Background: Young people in South Africa are at high risk of HIV infection and yet may have more limited access to prevention and treatment services than others in the population. Testing facilitates the sharing of prevention messages but also enables the linkage to care and treatment of those who test positive and therefore has wider public health implications.

Methods: This baseline survey conducted in 2005 for a community randomized trial in rural KwaZulu-Natal explored factors associated with a history of ever, repeat and recent testing amongst sexually debuted men and women aged 18 to 32 years.

Results: Over 35\% of this rural population ever tested for HIV, with men less likely to ever (unadjusted OR 0.26, 95\% Cl: 0.21-0.32) and repeatedly test than women (adjusted OR (aOR) 0.68, 95\% Cl: 0.48-0.97). Men aged 24-28 years (aOR 2.02, 95\% Cl: 1.10-3.71) and 29-32 years (aOR 2.69, 95\% Cl: 1.46-4.94) were more likely to ever test than those $<20$ years. Those who reported having discussed HIV with others had significantly greater odds of reporting ever (men's aOR 2.83, 95\% Cl: 1.63-4.89; women's aOR 3.36, 95\% Cl: 2.50-4.53), recent (irrespective of sex, aOR 2.87, 95\% Cl: 2.02-4.09) and repeat testing (aOR 2.02,95\% Cl: 1.28-3.19).
\end{abstract}

Conclusion: These findings highlight the need for novel youth- and men-friendly testing services and emphasises the importance of discussions about HIV in the home and community to encourage testing.

Keywords: HIV testing, Rural, South Africa, Young people

\section{Background}

South Africa has amongst the highest national adult HIV prevalence rate in the world, $12.3 \%$ in 2012 [1]. National survey data show that peak prevalence shifted in women from 25 to 29 years in 2008 to 30 to 35 years in 2012, possibly relating to increased life-expectancy resulting from antiretroviral treatment programmes [1]. Nonetheless, the burden of known new infections is largest amongst young people, particularly women under the age of 25 [2,3].

\footnotetext{
* Correspondence: Iknight@uwc.ac.za

${ }^{1}$ School of Public Health, University of the Western Cape, P Bag X17, Bellville 7535, South Africa

Full list of author information is available at the end of the article
}

Increasing access to and uptake of HIV testing among the general population, and especially young people, is a critical step in managing HIV and AIDS in South Africa. HIV testing has implications for prevention by encouraging risk reduction $[4,5]$ and for linkage to care [6]. Therefore the focus is increasingly on early and repeat testing, prior to symptomatic illness, either in voluntary or provider initiated counselling and testing (PICT) contexts. Despite a large national HIV testing campaign (2010-2011) and universal access to free testing through public health facilities, surveys show that fewer than $50 \%$ of adults know their status, with more than twice as many women reporting testing than men [1]. Prior to the campaign, younger age

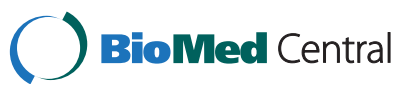


groups (15 to 25 years) [7-9] were least likely to have tested and fewer linked to medical care [10].

Studies in South Africa show evidence of high risk behaviours: two out of three of those who refuse testing reported HIV risk behaviour, including multiple sexual partners, exchanging sex for money, injecting drug usage and having had a sexually transmitted infections [11]. Despite the increased risk of HIV infection, perceptions of low risk exist amongst the general population [12] and young people $[13,14]$. This low risk perception is coupled with psychological barriers to testing, such as fear of the result, stigma and a perception that testing was for the symptomatic [8]. These and service-related barriers such as lack of confidentiality and distance to and delays at testing centres [15] account for low testing rates in South Africa. These barriers along with negative staff attitudes and inconvenient clinic hours, identified as key barriers to facility use by adolescents suggest an urgent need to address access to testing [16].

This paper provides insight into the HIV testing behaviour of young people aged 18 to 32 years prior to the implementation of a community-based trial. Few studies report on this broader age range, which reflects peak risk groups, such as women over 25, in South Africa [7,17]. Further, the uptake of HIV testing in rural communities is poorly reported on, with a greater focus on factors associated with HIV testing in urban and peri-urban contexts $[11,18,19]$. This study addresses these gaps by assessing the impact of various factors on ever, recent and repeat testing amongst 18-32 year old men and women in a high HIV prevalence, rural South African context.

\section{Methods}

This paper uses data from the baseline individual-level survey from the NIMH Project ACCEPT (HPTN 043) community randomized trial conducted in rural Vulindlela, KwaZulu-Natal (KZN) in 2005, similar analyses from the urban site have been published elsewhere [19]. This paper builds on the data published previously for the urban sites by providing a detailed analysis of the factors associated with testing in the rural South African context, and in a setting characterised by higher HIV prevelance. The HIV prevention trial included rural and urban communities in South Africa and communities in Tanzania, Zimbabwe and Thailand that were randomized to receive either a community-based HIV counselling and testing (HCT) intervention plus standard clinic-based HCT, or standard HCT alone. The intervention had three major strategies: (1) to make VCT more available in community settings (reduce barriers to testing; (2) to engage the community through outreach; and (3) to provide post-test support (reduce perceived adverse consequences of testing).
A random sample of households was selected from eight study communities, selected from within Vulindlela, with a combined population of approximately 40000 rural residents. In each selected household, one resident respondent, 18 to 32 years was randomly selected and enrolled. The total sample size was 2596.

All respondents provided informed consent and ethical clearance for the Project Accept study was provided by the University of California at Los Angeles South General Institutional Review Board and the University of the Witwatersrand.

To explore testing history we created three binary outcome variables: the first compared those who selfreported ever having an HIV test against those who do not report testing, the second those who self-reported having multiple tests against those who report ever testing once, and finally those self-reporting testing in the last six months, or recently, to those reporting ever testing. The baseline survey included systematically collected data about the reasons for testing for all those who ever tested.

The characteristics of the sample are described in Table 1. Recent behavioural characteristics indicative of increasing risk of HIV in the South African context were included to determine associations with recent testing, these included lower age at first sex, increased number of recent and lifetime sexual partners and no or inconsistent condom use. Indirect risk factors included in the analysis were self-reported drug and alcohol use which are sometimes associated with increased risk of HIV acquisition. Data on injecting drug use was collected but negligible and therefore excluded from the analysis and data about sexually transmitted infections was not collected. Variables were included that reflected individuals' attitudes to or perception of HIV. The first of these was a binary indicator for whether or not people report ever having had a discussion about HIV. This question was phrased in terms of a conversation with someone about HIV/AIDS. The respondent was also provided with a number of possible topics the conversation may have included including prevention, risk, testing and HIV and the community. This question was asked both in terms of lifetime discussions and discussions in the last 6 months. The question is limited in that it does not provide us with information about the timing or nature of the discussion. Secondly, two categorical variables were created based on summary means scores; one involved the six items used to measure social norms relating to HIV and the other comprised 22 items created to measure stigma associated with HIV [20]. Each was created using three categories: unfavourable, intermediate and favourable for social norms and low, intermediate or high levels of stigma towards HIV. The final variable was created using a score from questions about perceptions and knowledge of ART, this variable was also 
Table 1 Sample characteristics $(\mathrm{N}=2255)$ of sexually debuted $18-32$ year olds

\begin{tabular}{|c|c|c|c|c|c|}
\hline \multicolumn{3}{|l|}{ Characteristic } & \multirow{2}{*}{$\frac{\text { Male \% }(\mathbf{N}=\mathbf{9 2 3})}{18}$} & \multirow{2}{*}{$\frac{\text { Female } \%(N=1332)}{46}$} & \multirow{2}{*}{$\frac{\text { p-value }}{<0.001^{*}}$} \\
\hline Testing & Ever testing & Yes & & & \\
\hline & & No & 82 & 54 & \\
\hline & Recent testing & Yes & 7 & 17 & 0.41 \\
\hline & & No & 93 & 83 & \\
\hline & Repeat testing & Yes & 8 & 26 & $<0.001^{*}$ \\
\hline & & No & 92 & 74 & \\
\hline \multirow[t]{22}{*}{ Demographics } & Age & 18-20 years & 31 & 25 & $<0.001^{*}$ \\
\hline & & 21-24 years & 29 & 26 & \\
\hline & & $25-28$ years & 20 & 24 & \\
\hline & & $29-32$ years & 20 & 26 & \\
\hline & Household or individual & Low & 37 & 37 & 0.47 \\
\hline & Socio-economic status & Medium & 49 & 51 & \\
\hline & & High & 14 & 12 & \\
\hline & Years of education & $<7$ years & 8 & 10 & $0.02^{*}$ \\
\hline & & 8-10 years & 30 & 25 & \\
\hline & & $11-12$ years & 57 & 60 & \\
\hline & & Tertiary & 5 & 4 & \\
\hline & Occupation & Employed & 39 & 24 & $<0.001^{*}$ \\
\hline & & Unemployed & 32 & 59 & \\
\hline & & Student & 29 & 17 & \\
\hline & Income from work & Yes & 60 & 43 & $<0.001^{*}$ \\
\hline & & No & 40 & 57 & \\
\hline & Children under care & 0 & 67 & 24 & $<0.001^{*}$ \\
\hline & & 1 & 15 & 31 & \\
\hline & & $2+$ & 18 & 45 & \\
\hline & Cohabiting & Living apart & 25 & 18 & $<0.001^{*}$ \\
\hline & & No partner & 70 & 67 & \\
\hline & & Cohabiting & 6 & 15 & \\
\hline \multirow{15}{*}{$\begin{array}{l}\text { Behaviour indicative of increased } \\
\text { risk for HIV \& perceptions of HIV }\end{array}$} & No. of lifetime partners & 1 partner & 10 & 34 & $<0.001^{*}$ \\
\hline & & 2 partners & 14 & 30 & \\
\hline & & 3-4 partners & 25 & 28 & \\
\hline & & $5+$ partners & 51 & 8 & \\
\hline & Age at first sex & $<15$ years & 31 & 14 & $<0.001^{*}$ \\
\hline & & $16-17$ years & 37 & 36 & \\
\hline & & $18+$ years & 32 & 50 & \\
\hline & Lifetime alcohol & Yes & 80 & 29 & $<0.001^{*}$ \\
\hline & & No & 20 & 71 & \\
\hline & Lifetime drugs & Yes & 30 & 2 & $<0.001^{*}$ \\
\hline & & No & 70 & 98 & \\
\hline & Recent condom use & No partner & 26 & 22 & $<0.001^{*}$ \\
\hline & & Never & 26 & 37 & \\
\hline & & Sometimes & 20 & 17 & \\
\hline & & Always & 28 & 25 & \\
\hline
\end{tabular}


Table 1 Sample characteristics $(\mathbf{N}=\mathbf{2 2 5 5})$ of sexually debuted $18-32$ year olds (Continued)

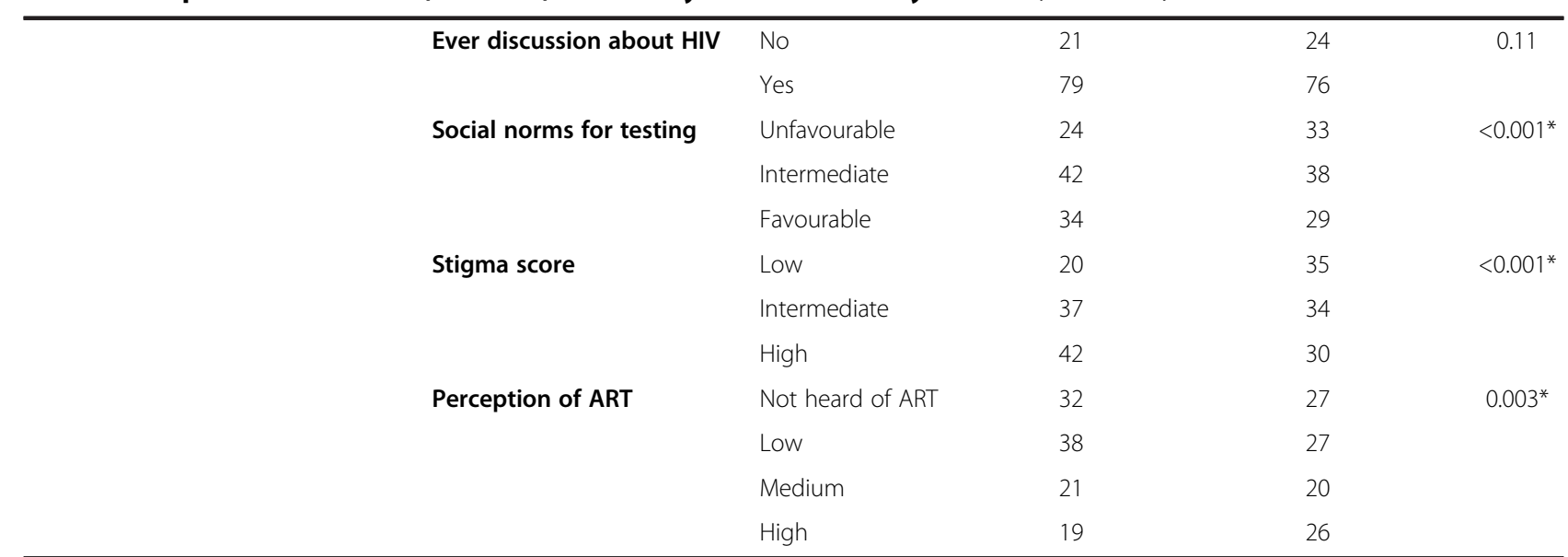

*Significant at the $95 \%$ confidence level.

categorical and included those who had not heard about ART and then three different levels (low, medium or high) of positive perception about ART.

The analyses considered only baseline respondents reporting ever having sex and therefore at risk of acquiring HIV. The three outcome variables were tested for univariate association with socio-demographic and behavioural characteristics associated with increased risk of HIV and perceptions of HIV. In order to better elucidate sex-specific characteristics associated with HIV testing, we present analyses of ever testing stratified by gender. For the multivariate analyses, age and gender (where appropriate) was included in all models a priori. Recent and repeat testing models were not split by gender because of the very small numbers reporting recent testing overall and men reporting repeat testing. All other variables were removed one at a time if inclusion did not significantly improve the fit of the model. Additional analyses present self-reported data about the most commonly selected reasons for choosing to have an HIV test (from a predefined list). These results provide insight into both the reason for testing. Analyses were conducted using STATA (STATACORP, version 11.0, College Station, TX) software.

\section{Results}

The analysis for this paper is based on 2,255 (89\% of total sample) young respondents who reported ever having sex. The majority of these sexually active respondents were women (59\%), reflecting the same proportion of women in the entire sample.

Table 1 shows that $35 \%$ of respondents reported ever having tested for HIV $(n=789)$, significantly more women reported ever $(46 \%)$ and repeat $(26 \%)$ testing than men ( $18 \%, \mathrm{p}=<0.001$ and $8 \%, \mathrm{p}=<0.001$ respectively). Of those who tested, $62 \%$ had last tested more than 6 months ago and $38 \%$ more recently. In terms of repeat testing, 53\% had tested more than once and $14 \%$ reported being HIVpositive. Men were significantly more likely to report behaviours that may be indicative of increased risk of HIV than women. Over $75 \%$ of men reported more than 3 lifetime sexual partners versus $34 \%$ for women. In addition men reported earlier sexual debut than women. Conversely, men seem to report more effective condom use in the six months prior to the interview than women, more men report not having a partner and always using a condom while fewer report never and only sometimes using condoms.

Of those reporting ever testing $84 \%$ gave one reason for testing, only $11 \%$ and one percent reported two or three reasons respectively. Table 2 shows the foremost reason for HIV testing was a desire to know one's status and the second was non-voluntary testing. Non-voluntary testing was self-defined but likely included PICT, antenatal routine testing or insurance testing. Neither testing because of ill health or sexual partner's risk were associated with gender. However, more women than men reported testing because of having children, non-voluntary testing and wanting to know their HIV status (for all $\mathrm{p}<0.01$; $100 \%, 98 \%$ and $74 \%$ of the total reporting these reasons were women).

Table 2 Ranking of most commonly cited reasons for choosing to have an HIV test $(\mathrm{N}=805)^{1}$

\begin{tabular}{lllll}
\hline & Total \% & Female \% & N & p-value $^{\mathbf{a}}$ \\
\hline Wanted to know status & 51 & 74 & 408 & $0.001^{*}$ \\
Non-voluntary & 19 & 98 & 149 & $<0.001^{*}$ \\
Having children & 17 & 100 & 137 & $<0.001^{*}$ \\
Sick & 12 & 75 & 95 & 0.31 \\
$\begin{array}{l}\text { Sexual partner had } \\
\text { risky behaviour }\end{array}$ & 0.03 & 89 & 26 & 0.19
\end{tabular}

${ }^{\mathrm{a}}$ Chi-square tests for comparison of men and women.

*Significant at the $95 \%$ confidence level.

${ }^{1}$ The questions for Table 2 allow for multiple responses so the $\mathrm{N}$ may overlap as discussed in the text. The total $\mathrm{N}$ is 805 . 


\section{HIV testing among men}

Of the 923 sexually active male participants, only 170 (18\%) reported ever testing for HIV. A significant association exists between ever testing and age; men over 25 years have increased odds of ever testing for HIV (Table 3). In the adjusted model, there was no significant association between ever reporting HIV testing and education, socioeconomic status or number of children under care. There was an association between testing and men's occupation with male students having reduced odds of testing. Fewer than $4 \%(n=3)$ were tested at their workplace and therefore this is unlikely to have influenced this finding.

No risk behaviour variables were associated with ever testing in men in the adjusted model. Neither were stigma, social norms or perceptions of ART. Discussions about HIV were significantly associated with ever testing, men who had had discussions were almost three times more likely to have ever tested (aOR 2.83, 95\% CI: 1.63-4.89) compared to those men who had not discussed HIV.

\section{HIV testing among women}

Of the 1,332 sexually active women in the study, 619 (46\%) reported ever testing for HIV. Age was associated with reporting ever testing, women over 25 years had lower odds of testing (Table 3). Occupation was significantly associated with testing among women in a univariable model but was not significant in a multivariable model adjusted for age and those factors associated in the univariable. Women with children under their care had higher odds of ever testing than those with no children in the adjusted model.

Like men, no behavioural risk factors nor stigma or social norms were associated with ever testing among women. Women reporting a discussion about HIV were more than three times more likely to have ever tested compared with those who did not have a discussion (aOR 3.36, 95\% CI: 2.50-4.53). Women reporting a high perception of ART had higher odds of reporting HIV testing (aOR 1.63, 95\% CI: 1.17-2.26) relative to women who had not heard of ART.

\section{Recent and repeat testing}

Table 4 shows the factors associated with recent and repeat testing in both an unadjusted and adjusted model. The adjusted model shows that unlike ever testing, no difference exists by gender for recent testing, although women were more likely to repeat test. Those older than 25 had lower odds of recent testing than other age groups, but no association exists between age and repeat testing. Employed people had lower odds of recent testing than the unemployed. In contrast socio-economic status is associated with repeat testing only, those with low and high socio-economic status have higher odds of repeat testing than those in the middle category.
Unlike ever testing among women, that showed a relationship between having children and ever testing, no other socio-demographic variables significantly affected the final models for recent or repeat testing.

While no risk factors were associated with ever or recent testing, frequency of condom use was associated with repeat testing. Those reporting no recent sexual partners or no or infrequent recent condom use had lower odds of repeat testing for HIV compared to those always using condoms.

Neither social norms nor stigma were associated with recent or repeat testing. In contrast, those with no knowledge or a low or medium positive perception of ART all had lower odds of repeat testing than those with high positive perception. Similar to findings for ever testing, those who report a discussion about HIV had significantly greater odds of both recent and repeat testing compared to those who reported no discussions.

\section{Discussion}

In this representative baseline sample of sexually active 18-32-year-olds from rural KwaZulu-Natal, 35\% of respondents report ever testing for HIV in 2005, with gender, age and discussions about HIV emerging as important factors in determining HIV testing.

Studies show poor uptake of testing in rural populations in South Africa [9,21]. This is particularly concerning in high HIV prevalence contexts such as KZN [21]. Despite using the same methodology and a similar sample, HIV testing in this rural community is lower than in the Soweto (urban) Project Accept site (48\%) over the same period [19]. Alternative models of testing are therefore required to ensure the maximum testing coverage in rural populations.

Gendered patterns of testing demonstrate that men had lower rates of ever and repeat testing than women. Similar gender differential were observed in a 2003 national survey of youth 15 to 24 years, although overall rates of testing are lower than observed here, possibly because of lower testing rates in men under 24 [17]. Gendered patterns of testing highlight the need to increase testing opportunities for men, especially younger men.

Motivations for testing are also gendered with significantly more women reporting non-voluntary and antenatal testing as opportunities to test. This may explain the reduced rate of testing in women without children. Evidence from the urban site and others demonstrates that, in addition to PICT in pregnancy, women's motivations may be linked to the perceived benefits for their infant [19,22-24]. It is essential to give all women the opportunity to know their status and address their risks for HIV [17].

The gender imbalance in both testing and motivations is compounded by evidence from sub-Saharan Africa that women have more contact with health facilities than 
Table 3 Socio-demographic factors, recent indicators of behavioural risk and perceptions of HIV associated with reporting ever testing amongst men and women

\begin{tabular}{|c|c|c|c|c|c|c|c|c|c|c|}
\hline & & \multirow[b]{2}{*}{ Characteristic } & \multicolumn{3}{|c|}{ Men N = 923} & \multirow[b]{2}{*}{$\begin{array}{l}\text { LRT } \\
p \text { value }\end{array}$} & \multicolumn{3}{|c|}{ Women $\mathrm{N}=1332$} & \multirow[b]{2}{*}{$\begin{array}{l}\text { LRT } \\
\text { p value }\end{array}$} \\
\hline & & & $\begin{array}{l}\text { Test } \% \\
(n=170)\end{array}$ & $\begin{array}{l}\text { Unadjusted OR } \\
(95 \% \mathrm{Cl})\end{array}$ & $\begin{array}{l}\text { Adjusted OR* } \\
\text { (95\% Cl) }\end{array}$ & & $\begin{array}{c}\text { Test } \% \\
(n=619)\end{array}$ & $\begin{array}{c}\text { Unadjusted OR } \\
(95 \% \mathrm{Cl})\end{array}$ & $\begin{array}{l}\text { Adjusted OR** } \\
(95 \% \mathrm{Cl})\end{array}$ & \\
\hline \multirow[t]{22}{*}{ Demographics } & Age & $18-20$ years & 15 & 1 & 1 & 0.01 & 27 & 1 & 1 & $<0.001$ \\
\hline & & 21-24 years & 29 & $2.21(1.33-3.69)$ & $1.67(0.96-2.93)$ & & 29 & $1.07(0.79-1.45)$ & $0.86(0.62-1.21)$ & \\
\hline & & $25-28$ years & 25 & $2.95(1.74-5.00)$ & $2.02(1.10-3.71)$ & & 22 & $0.75(0.55-1.02)$ & $0.55(0.39-0.69)$ & \\
\hline & & 29-32 years & 31 & $4.02(2.40-6.73)$ & $2.69(1.46-4.94)$ & & 23 & $0.67(0.49-0.91)$ & $0.48(0.33-0.69)$ & \\
\hline & Socio-economic status & Low & 34 & $0.85(0.59-1.22)$ & & & 38 & $1.13(0.89-1.42)$ & & \\
\hline & & Medium & 51 & 1 & & & 50 & 1 & & \\
\hline & & High & 15 & $1.04(0.63-1.71)$ & & & 11 & $0.91(0.64-1.28)$ & & \\
\hline & Years of education & $<7$ years & 5 & $0.54(0.26-1.13)$ & & & 11 & $1.26(0.88-1.82)$ & & \\
\hline & & $8-10$ years & 24 & $0.67(0.45-0.99)$ & & & 27 & $1.24(0.96-1.60)$ & & \\
\hline & & $11-12$ years & 62 & 1 & & & 57 & 1 & & \\
\hline & & Tertiary & 8 & $1.78(0.91-3.46)$ & & & 5 & $1.35(0.79-2.31)$ & & \\
\hline & Occupation & Employed & 38 & 1 & 1 & 0.06 & 21 & 1 & 1 & $<0.001$ \\
\hline & & Unemployed & 48 & $0.94(0.65-1.36)$ & $1.03(0.71-1.51)$ & & 63 & $1.45(1.12-1.89)$ & $1.26(0.96-1.66)$ & \\
\hline & & Student & 14 & $0.31(0.19-0.51)$ & $0.53(0.29-0.96)$ & & 15 & $1.02(0.72-1.44)$ & $0.77(0.51-1.16)$ & \\
\hline & Income from work & Yes & 66 & 1 & & & 41 & 1 & & \\
\hline & & No & 34 & $0.70(0.49-0.98)$ & & & 59 & $1.15(0.92-1.43)$ & & \\
\hline & Children under care & 0 & 62 & 1 & & & 18 & 1 & 1 & $<0.001$ \\
\hline & & 1 & 20 & $1.50(0.97-2.33)$ & & & 34 & $1.06(1.52-2.78)$ & $2.11(1.55-2.88)$ & \\
\hline & & $2+$ & 18 & $1.08(0.69-1.69)$ & & & 48 & $1.83(1.38-2.43)$ & $2.00(1.49-2.69)$ & \\
\hline & Cohabiting & Living apart & 75 & 1 & & & 67 & 1 & & \\
\hline & & No partner & 18 & $0.63(0.41-0.97)$ & & & 17 & $0.86(0.65-1.15)$ & & \\
\hline & & Cohabiting & 6 & $1.11(0.55-2.22)$ & & & 16 & $1.18(0.87-1.61)$ & & \\
\hline \multirow{7}{*}{$\begin{array}{l}\text { Behaviour indicative of } \\
\text { increased risk for HIV \& } \\
\text { Perceptions of HIV }\end{array}$} & No. of lifetime partners & 1 partner & 8 & $0.57(0.31-1.07)$ & & & 35 & $0.82(0.53-1.26)$ & & \\
\hline & & 2 partners & 39 & $0.36(0.20-0.67)$ & & & 28 & $0.70(0.45-1.08)$ & & \\
\hline & & 3-4 partners & 20 & $0.57(0.37-0.87)$ & & & 28 & $0.80(0.51-1.24)$ & & \\
\hline & & $5+$ partners & 65 & 1 & & & 9 & 1 & & \\
\hline & Age at first sex & $<15$ years & 33 & $0.97(0.64-1.45)$ & & & 16 & $1.35(0.97-1.88)$ & & \\
\hline & & $16-17$ years & 32 & $0.76(0.51-1.15)$ & & & 36 & $1.02(0.81-1.29)$ & & \\
\hline & & $18<$ years & 35 & 1 & & & 49 & 1 & & \\
\hline
\end{tabular}


Table 3 Socio-demographic factors, recent indicators of behavioural risk and perceptions of HIV associated with reporting ever testing amongst men and women (Continued)

\begin{tabular}{|c|c|c|c|c|c|c|c|c|c|}
\hline Lifetime alcohol & Yes & 76 & 1 & & & 31 & 1 & & \\
\hline & No & 24 & $1.34(0.90-1.99)$ & & & 69 & $0.85(0.67-1.08)$ & & \\
\hline Lifetime drugs & Yes & 31 & $1.02(0.71-1.47)$ & & & 2 & $1.63(0.72-3.69)$ & & \\
\hline & No & 69 & 1 & & & 98 & 1 & & \\
\hline Ever discussion about HIV & No & 10 & 1 & 1 & $<0.001$ & 87 & 1 & 1 & $<0.001$ \\
\hline & Yes & 90 & $2.83(1.67-4.80)$ & $2.83(1.63-4.89)$ & & 13 & $3.34(2.53-4.42)$ & $3.36(2.50-4.53)$ & \\
\hline Social norms for testing & Unfavourable & 27 & $1.40(0.90-2.19)$ & & & 31 & $0.93(0.70-1.22)$ & & \\
\hline & Intermediate & 44 & $1.32(0.89-1.96)$ & & & 40 & $1.14(0.87-1.48)$ & & \\
\hline & Favourable & 29 & 1 & & & 29 & 1 & & \\
\hline Stigma Score & Low & 22 & $1.22(0.78-1.90)$ & & & 34 & $0.87(0.67-1.14)$ & & \\
\hline & Intermediate & 38 & $1.08(0.74-1.57)$ & & & 34 & $0.91(0.69-1.19)$ & & \\
\hline & High & 40 & 1 & & & 32 & 1 & & \\
\hline Perception of ART & Not heard of ART & 21 & 1 & & & 22 & 1 & 1 & $<0.001$ \\
\hline & Low & 36 & $2.20(1.40-3.46)$ & & & 28 & $1.43(1.06-1.92)$ & $1.18(0.86-1.63)$ & \\
\hline & Medium & 22 & $1.68(1.02-2.76)$ & & & 19 & $1.28(0.93-1.77)$ & $1.00(0.71-1.41)$ & \\
\hline & High & 21 & $1.75(1.05-2.90)$ & & & 31 & $2.03(1.50-2.74)$ & $1.63(1.17-2.26)$ & \\
\hline
\end{tabular}

*Model is adjusted for Age, Occupation, and Report of a discussion about HIV.

**Model is adjusted for Age, Occupation, Children under care, Report of a discussion about HIV and Perception of ART.

Bold indicates Significant at the $95 \%$ confidence level. 
Table 4 Socio-demographic factors, recent indicators of behavioural risk and perceptions of HIV associated with recent (last 6 months) and repeated testing for HIV amongst those ever testing ( $N=789)$

\begin{tabular}{|c|c|c|c|c|c|c|c|c|c|c|}
\hline Characteristic & & & $\begin{array}{l}\text { Recent Test \% } \\
\quad(n=296)\end{array}$ & $\begin{array}{c}\text { Unadjusted OR } \\
\quad(95 \% \mathrm{Cl})\end{array}$ & $\begin{array}{l}\text { Adjusted OR* } \\
(95 \% \mathrm{Cl})\end{array}$ & $\begin{array}{c}\text { LRT } \\
\text { p value }\end{array}$ & $\begin{array}{c}\text { Repeated \% } \\
(n=417)\end{array}$ & $\begin{array}{l}\text { Unadjusted OR } \\
(95 \% \mathrm{Cl})\end{array}$ & $\begin{array}{l}\text { Adjusted OR }{ }^{* *} \\
(95 \% \mathrm{Cl})\end{array}$ & $\begin{array}{l}\text { LRT } \\
\text { p value }\end{array}$ \\
\hline \multirow[t]{24}{*}{ Demographics } & \multirow[t]{2}{*}{ Gender } & Male & 21 & $0.94(0.66-1.34)$ & $1.19(0.81-1.74)$ & $<0.001$ & 18 & $0.68(0.48-0.95)$ & $0.68(0.48-0.97)$ & 0.02 \\
\hline & & Female & 79 & 1 & 1 & & 82 & 1 & 1 & \\
\hline & \multirow[t]{4}{*}{ Age } & $18-20$ years & 29 & 1 & 1 & 0.10 & 26 & 1 & 1 & \\
\hline & & 21-24 years & 30 & $0.79(0.53-1.16)$ & $0.77(0.51-1.18)$ & & 28 & $0.82(0.56-1.21)$ & $0.89(0.60-1.34)$ & \\
\hline & & $25-28$ years & 20 & $0.59(0.38-0.89)$ & $0.61(0.38-0.98)$ & & 23 & $0.85(0.56-1.28)$ & $0.81(0.52-1.24)$ & \\
\hline & & 29-32 years & 21 & $0.60(0.39-0.90)$ & $0.61(0.38-0.97)$ & & 24 & $0.80(0.54-1.20)$ & $0.76(0.50-1.16)$ & \\
\hline & \multirow[t]{3}{*}{ Socio-economic status } & Low & 37 & $0.99(0.72-1.35)$ & & & 41 & $1.51(1.11-2.05)$ & $1.49(1.10-2.02)$ & 0.01 \\
\hline & & Medium & 50 & 1 & & & 46 & 1 & 1 & \\
\hline & & High & 13 & $1.07(0.67-1.69)$ & & & 13 & $1.57(0.999-2.47)$ & $1.61(1.02-2.54)$ & \\
\hline & \multirow[t]{4}{*}{ Years of education } & $<7$ years & 11 & $1.32(0.81-2.15)$ & & & 12 & $1.51(0.92-2.48)$ & & \\
\hline & & 8-10 years & 29 & $1.23(0.88-1.72)$ & & & 25 & $0.93(0.67-1.29)$ & & \\
\hline & & 11-12 years & 56 & 1 & & & 58 & 1 & & \\
\hline & & Tertiary & 4 & $0.59(0.29-1.19)$ & & & 6 & $1.10(0.59-2.04)$ & & \\
\hline & \multirow[t]{3}{*}{ Occupation } & Employed & 19 & $0.49(0.34-0.70)$ & $0.50(0.34-0.73)$ & $<0.001$ & 27 & 1 & & \\
\hline & & Unemployed & 65 & 1 & 1 & & 57 & $1.02(0.74-1.41)$ & & \\
\hline & & Student & 17 & $1.03(0.68-1.55)$ & $0.86(0.55-1.34)$ & & 16 & $1.21(0.80-1.82)$ & & \\
\hline & \multirow[t]{2}{*}{ Income from work } & Yes & 41 & $0.68(0.51-0.91)$ & & & 48 & 1 & & \\
\hline & & No & 59 & 1 & & & 52 & $0.89(0.67-1.18)$ & & \\
\hline & \multirow[t]{3}{*}{ Children under care } & 0 & 31 & $1.31(0.92-1.87)$ & & & 24 & $0.69(0.49-0.98)$ & & \\
\hline & & 1 & 29 & $0.95(0.67-1.35)$ & & & 31 & $0.88(0.63-1.23)$ & & \\
\hline & & $2+$ & 38 & 1 & & & 44 & 1 & & \\
\hline & \multirow[t]{3}{*}{ Cohabiting } & No partner & 18 & $1.10(0.74-1.62)$ & & & 18 & $1.06(0.72-1.54)$ & & \\
\hline & & Living apart & 66 & 1 & & & 69 & 1 & & \\
\hline & & Cohabiting & 16 & 1.27 (0.84-1.92) & & & 13 & $0.81(0.54-1.22)$ & & \\
\hline \multirow{7}{*}{$\begin{array}{l}\text { Behaviour indicative of } \\
\text { increased risk for HIV \& } \\
\text { Perceptions of HIV }\end{array}$} & \multirow{3}{*}{ Recent sexual partners } & 0 Partners & 21 & $1.07(0.75-1.53)$ & & & 21 & $1.09(0.76-1.54)$ & & \\
\hline & & 1 Partner & 72 & 1 & & & 72 & 1 & & \\
\hline & & $2+$ Partners & 7 & $0.93(0.53-1.62)$ & & & 7 & $0.80(0.47-1.38)$ & & \\
\hline & \multirow[t]{4}{*}{ Recent condom use } & No partners & 21 & $0.93(0.61-1.42)$ & & & 21 & $0.80(0.53-1.21)$ & $0.80(0.52-1.23)$ & 0.04 \\
\hline & & Never & 29 & $0.76(0.52-1.12)$ & & & 30 & $0.63(0.43-0.91)$ & $0.62(0.42-0.91)$ & \\
\hline & & Sometimes & 22 & $0.99(0.64-1.49)$ & & & 18 & $0.58(0.39-0.88)$ & $0.57(0.37-0.88)$ & \\
\hline & & Always & 28 & 1 & & & 30 & 1 & & \\
\hline
\end{tabular}


Table 4 Socio-demographic factors, recent indicators of behavioural risk and perceptions of HIV associated with recent (last 6 months) and repeated testing for HIV amongst those ever testing ( $N=789)$ (Continued)

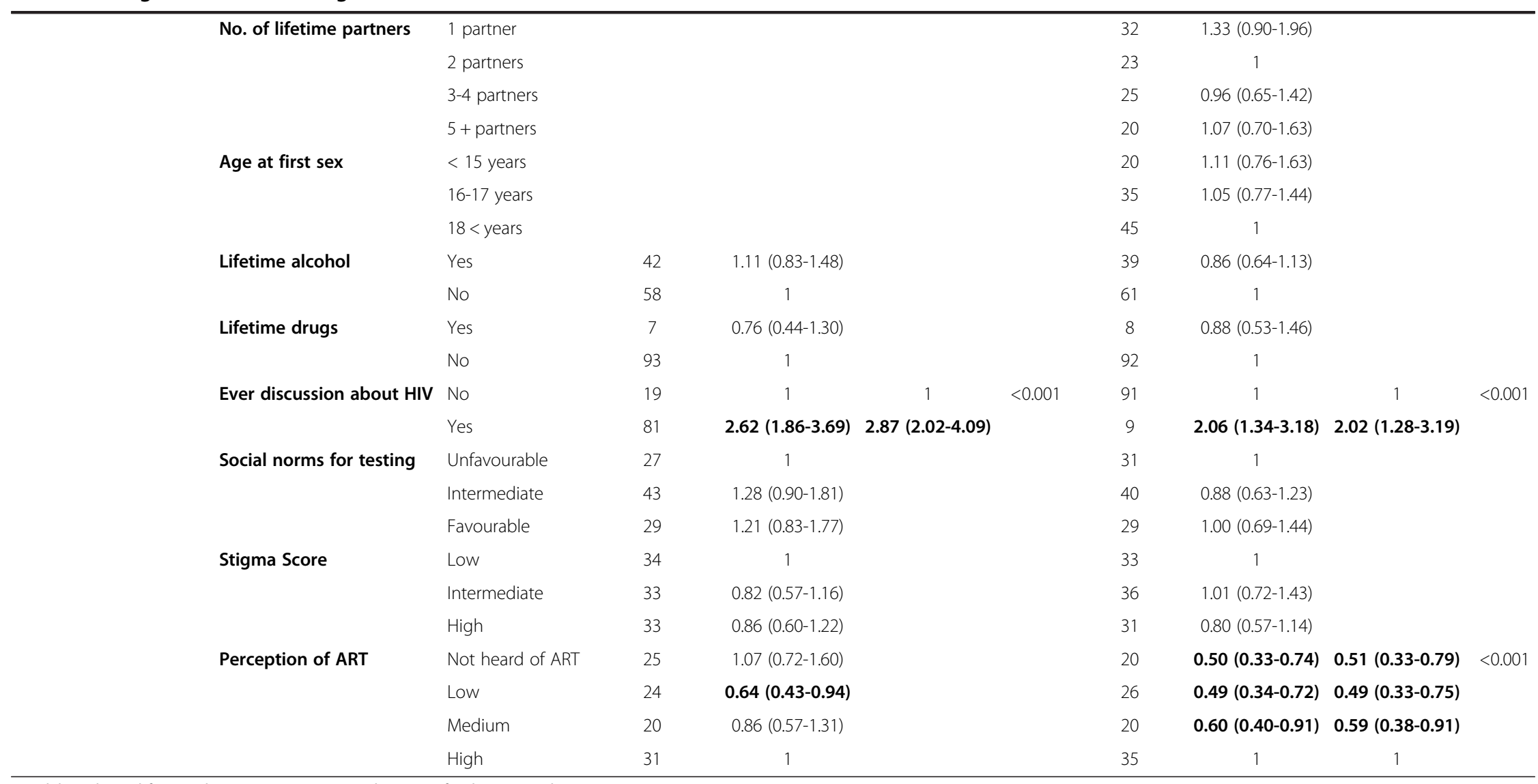

**Model is adjusted for Gender, Age, Socio-economic status, Recent condom use, Report of a discussion about HIV and Perception of ART.

Bold indicates Significant at the $95 \%$ confidence level. 
men, mainly through reproductive and child health services $[25,26]$. Moreover, health services are not considered male-friendly spaces, with operating hours and provider attitudes that may lack sensitivity to men's needs [27]. Consequently, fewer men than women are establishing and disclosing their HIV status [28], acknowledging their symptoms, and accessing treatment across Africa [29-31].

Lower testing rates among younger men in this high prevalence context are a concern. Despite lower rates of HIV than among women of a similar age [1], men reported higher rates of risk behaviours than women, making them an important target group for testing, risk awareness and prevention messaging. Young men experience similar barriers to health service access as older men $[8,32,33]$, highlighting the need for more acceptable and accessible services for all men [19,34].

The association between conversations about HIV and testing is noted in both national and urban South African studies of young people $[17,19]$. A joint analysis of the South African Project Accept data [35] shows findings similar to those observed here with discussions associated with a significantly increased odds of testing (between 1.29 to 3 times). To account for the influence of reverse causality, an analysis including both recent testing and discussions observes that the relationship still exists. While the nature of these discussions or with whom they took place is hard to discern, evidence suggests that discussions about HIV play a role in the decision to test, possibly through normalising HIV and reducing the fear associated with testing.

The observed association between a positive perception of ART and ever testing in women and repeat testing is corroborated by qualitative data from the same period and communities showing that access to ART influences the decision to test for HIV [36]. Again, causality is not implied because ART access may follow testing. However, the association is also supported by evidence from a rural KZN cohort where access to ART increased over time (2005 to 2011) alongside knowledge of HIV status [37].

Although, no relationship was observed between stigma and testing, the relative importance of access to ART coupled with the influence of discussions on testing has the potential to shift risk perceptions and stigma by encouraging disclosure and conversations about HIV amongst individuals, couples, families and communities.

Except for a relationship between repeat testing and recent condom use evidence for associations between risk and testing do not exist in this and the urban data [19]. The observed association suggests a reduction in risk behaviours because of repeat testing or that cautious people test more often. A rural HIV survey of young people 15-24 years (2006 to 2011) demonstrated a relationship between testing and reduced HIV incidence likely due to behaviour change and the potential positive impact of testing in this context [38].

While there was minimal evidence for association between socio-economic variables and testing, employed people did show a lower odds of recent testing than the unemployed, this is possibly linked to access to testing which is traditionally provided in public facilities during work hours and not always easy for those with employment to access. This issue of access may also be related to the observed relationship between socio-economic status and repeat testing that suggests that those with low and high socio-economic status have higher odds of repeat testing, possibly because of access to free public testing by those with low status and the ability of those with higher status to afford alternatives to free public testing. This highlights the need to provide alternatives to standard opportunities for testing everyone for free.

\section{Limitations}

The data from this paper was collected in 2005 and it is likely that the situation has changed. At the time, PICT was not policy and ART availability was not widespread. Certainly this and other research shows that increased access to treatment and its normalisation influence testing outcomes [36]. In addition, the National HIV testing campaign has increased general access to and uptake of testing, nonetheless these findings suggest that there is a need for testing and in particular a focus on recent and repeat testing [6].

The results regarding testing, stigma and behaviours associated with increased risk for HIV may have been influenced by social desirability bias.

The data about discussions about HIV are limited because we lack information about the timing, nature and parties involved in these conversations. Although the inclusion of the recent testing analysis with information about a recent discussion tries to respond to the problem of reverse causality the data is still limited. The variable is included in the analysis because despite these potential limitations it highlights the importance of converstaions around HIV and testing and points to possible avenues for further exploration and intervention research.

\section{Conclusion}

The provision of male and youth appropriate HIV testing services is required to deliver HCT services at scale in this high prevalence, rural context. HCT provides an opportunity to emphasise prevention and to link positive people to care and treatment thereby reducing infectiousness and disease spread [39]. Effective control of the epidemic in South Africa requires massive scale up of alternate models of HCT alongside facility-based testing to provide targeted and responsive services to women, men and young people. Strong evidence exists 
that community-based HCT (mobile and home-based) approaches are capable of reaching a wider range of target groups with coverage in both rural and urban locations and to address the convenience factors typically associated with health facility-based HCT [40-43] in sub-Saharan Africa. These particular target groups may require even more innovative approaches to testing and access though to ensure they are reached. Targeting these and other hard to reach key populations not reflected in this research requires the provision of multiple options and a range of approaches for testing, in order to maximise the opportunities to test and engage with services. Alternative options for accessing testing that bypasses many of the acknowledged barriers may include offering testing in work and social spaces where men in particular gather. Another opportunity for testing that has the potential to create opportunities for potential disclosure in a supportive environment is couples testing [40]. Although not currently available preliminary research suggests that self-testing may present welcome opportunities for testing target groups that struggle to access facility-based testing [41,42].

Men and younger people should also be targeted through these methods earlier and younger, school-based testing provides an opportunity to test young people easily, but there is a need to overcome political and social difficulties that affect school-based testing and has remained a challenge in the South African system to date. These should be widely implemented alongside other models of testing to facilitate uptake in South Africa [43]. The role of discussions in influencing HIV testing holds promise for its potential in shifting norms about risk perception, treatment and ultimately stigma and discrimination in this context.

\section{Competing interests}

The authors declare that they have no competing interests.

\section{Authors' contributions \\ All authors were involved in conceiving the research question. LK designed and conducted the analysis and wrote the manuscript. NM contributed to the interpretation of the data analysis and reviewed the manuscript. HvR, $\mathrm{HH}, \mathrm{AvH}$ reviewed the manuscript. LR reviewed the manuscript and is a Principal Investigator. LK had primary responsibility for final content. All authors read and approved the final manuscript.}

\section{Acknowledgements}

This research was sponsored by the U.S. National Institute of Mental Health as a cooperative agreement, through contracts U01MH066687 (Johns Hopkins University - David Celentano, PI); U01MH066688 (Medical University of South Carolina - Michael Sweat, PI); U01MH066701 (University of California, Los Angeles - Thomas J. Coates, PI); and U01MH066702 (University of California, San Francisco - Stephen F. Morin, PI). In addition, this work was supported as HPTN Protocol 043 through contracts U01Al068613/UM1AI068613 (HPTN Network Laboratory - Susan Eshleman, PI); U01Al068617/UM1Al068617 (SCHARP - Deborah Donnell, PI); and U01Al068619/UM1AI068619 (HIV Prevention Trials Network - Sten Vermund/Wafaa El-Sadr, PIs) of the Division of AIDS of the U.S. National Institute of Allergy and Infectious Diseases; and by the Office of AIDS Research of the U.S. National Institutes of Health. Views expressed are those of the authors, and not necessarily those of sponsoring agencies. Nuala McGrath was supported by a Wellcome Trust fellowship (grant \# WT083495MA).

\section{Author details}

'School of Public Health, University of the Western Cape, P Bag X17, Bellville 7535 , South Africa. ${ }^{2}$ Academic Unit of Primary Care and Population Sciences, and Department of Social Statistics and Demography, Mailpoint 805,

Southampton General Hospital, Tremona Road, Southampton SO16 6YD, UK. ${ }^{3}$ Africa Centre for Health and Population Studies, University of KwaZulu-Natal, PO Box 198, Mtubatuba KZN 3935, South Africa. ${ }^{4}$ HIV/AIDS STIs and TB Programme, Human Sciences Research Council, P.O Box 90, Msunduzi 3200, South Africa. ${ }^{5}$ Developmental Pathways of Health Research Unit, Department of Paediatrics, School of Clinical Medicine, Faculty of Health Sciences, University of the Witwatersrand, Johannesburg, South Africa. ${ }^{6}$ HIV/AIDS STIs and TB Programme, Human Sciences Research Council, 750 Francois Road, Cato Manor, Durban 4001, South Africa. 'DST-NRF Centre of Excellence in Human Development, University of the Witwatersrand, Johannesburg, South Africa.

Received: 8 July 2014 Accepted: 17 October 2014 Published: 13 November 2014

\section{References}

1. Shisana O, Rehle T, Simbayi L, Zuma K, Jooste S, Zungu N, Labadarios D, Onoya D, Davids A, Ramlagan S, Van Zyl J, Mbelle N, Wabiri N: South African National HIV Prevalence, Incidence and Behaviour Survey, 2012. Cape Town: 2014.

2. Abdool Karim Q, Kharsany ABM, Frohlich J a, Werner L, Mlotshwa M, Madlala BT, Abdool Karim SS: HIV incidence in young girls in KwaZulu-Natal, South Africa-public health imperative for their inclusion in HIV biomedical intervention trials. AIDS Behav 2012, 16:1870-1876.

3. Shisana O, Rehle T, Simbayi LC, Zuma K, Jooste S, Pillay-van-Wyk V, Mbelle N, Van Zyl J, Parker W, Pezi S: Team the Sll: South African National HIV Prevalence, Incidence, Behaviour and Communication Survey, 2008 A Turning Tide Among Teenagers?. Cape Town: HSRC Press; 2009.

4. Delavande A, Kohler H-P: The impact of HIV testing on subjective expectations and risky behavior in Malawi. Demography 2012, 49:1011-1036.

5. King R, Lifshay J, Nakayiwa S, Katuntu D, Lindkvist P, Bunnell R: The virus stops with me: HIV-infected Ugandans' motivations in preventing HIV transmission. Soc Sci Med 2009, 68:749-757.

6. Department of Health: South African National HIV Counselling And Testing (HCT) Policy Guidelines. Pretoria: South African National Department of Health; 2010.

7. Hutchinson PL, Mahlalela X: Utilization of voluntary counseling and testing services in the Eastern Cape, South Africa. AIDS Care 2006, 18:446-455.

8. MacPhail CL, Pettifor A, Coates T, Rees H: "You must do the test to know your status": attitudes to HIV voluntary counseling and testing for adolescents among South African youth and parents. Heal Educ Behav 2008, 35:87-104.

9. Peltzer K, Matseke G, Mzolo T, Majaja M: Determinants of knowledge of HIV status in South Africa: results from a population-based HIV survey. BMC Public Health 2009, 9:174.

10. Liechty CA: The evolving role of HIV counseling and testing in resourcelimited settings: HIV prevention and linkage to expanding HIV care access. Curr Infect Dis Rep 2005, 7:154-158.

11. Kalichman SC, Simbayi LC: HIV testing attitudes, AIDS stigma, and voluntary HIV counselling and testing in a black township in Cape Town, South Africa. Sex Transm Infect 2003, 79:442-447.

12. Cherutich P, Bunnell R, Mermin J: HIV testing: current practice and future directions. Curr HIV/AIDS Rep 2013, 10:134-141.

13. Pettifor A, Macphail C, Rees H, Cohen M: HIV and sexual behavior among young people: the South African paradox. Sex Transm Dis 2008, 35:843-844.

14. Myburgh H: The Clinic as a Gendered Space: Masculinities, Health Seeking Behavior and HIV \& AIDS. Johannesburg: Consultancy Africa Intelligence; 2011:1-5 [Public Health- Discussion Papers].

15. Matovu JKB, Makumbi FE: Expanding access to voluntary HIV counselling and testing in sub-Saharan Africa: alternative approaches for improving uptake, 2001-2007. Trop Med Int Heal 2007, 12:1315-1322.

16. Pettifor AE, Measham DM, Rees HV, Padian NS: Sexual power and HIV risk, South Africa. Emerg Infect Dis 2004, 10:1996-2004.

17. MacPhail C, Pettifor A, Moyo W, Rees H: Factors associated with HIV testing among sexually active South African youth aged 15-24 years. AIDS Care 2009, 21:456-467. 
18. Pettifor A, MacPhail C, Suchindran S, Delany-Moretlwe S: Factors associated with HIV testing among public sector clinic attendees in Johannesburg, South Africa. AlDS Behav 2010, 14:913-921.

19. Venkatesh KK, Madiba P, de Bruyn G, Lurie MN, Coates TJ, Gray GE: Who gets tested for HIV in a South African urban township? Implications for test and treat and gender-based prevention interventions. JAIDS 2011 56:151-165

20. Genberg BL, Kawichai S, Chingono A, Sendah M, Chariyalertsak S, Konda KA, Celentano DD: Assessing HIV/AIDS stigma and discrimination in developing countries. AIDS Behav 2008, 12:772-780.

21. Tabana H, Doherty T, Swanevelder S, Lombard C, Jackson D, Zembe W, Naik R: Knowledge of HIV status prior to a community HIV counseling and testing intervention in a rural district of South Africa: results of a community based survey. BMC Infect Dis 2012, 12:73.

22. Perez F, Zvandaziva C, Engelsmann B, Dabis F: Acceptability of routine HIV testing ("opt-out") in antenatal services in two rural districts of Zimbabwe. J Acquir Immune Defic Syndr 2006, 41:514-520.

23. Etiebet M, Fransman D, Forsyth B: Integrating prevention of mother-to-child HIV transmission into antenatal care: learning from the experiences of women in South Africa. AIDS Care 2004, 16:37-46.

24. Obermeyer CM, Osborn M: The utilization of testing and counseling for HIV: a review of the social and behavioral evidence. Am J Public Health 2007, 97:1762-1774.

25. Snow RC, Madalane M, Poulsen M: Are men testing? Sex differentials in HIV testing in Mpumalanga Province, South Africa. AIDS Care 2010, 22:1060-1065

26. Mills EJ, Beyrer C, Birungi J, Dybul MR: Engaging men in prevention and care for HIV/AIDS in Africa. PLoS Med 2012, 9:e1001167.

27. Koo K, Makin JD, Forsyth BWC: Barriers to male-partner participation in programs to prevent Mother-to-Child HIV transmission in South Africa. AIDS Educ Prev 2013, 25:14-24.

28. Dageid W, Govender K, Gordon SF: Masculinity and HIV disclosure among heterosexual South African men: implications for HIV/AIDS intervention. Cult Health Sex 2012, 14:925-940.

29. Cornell M, Mclntyre J, Myer L: Men and antiretroviral therapy in Africa: our blind spot. Trop Med Int Health 2011, 16:828-829.

30. Muula AS, Ngulube TJ, Siziya S, Makupe CM, Umar E, Prozesky HW, Wiysonge $\mathrm{CS}$, Mataya $\mathrm{RH}$ : Gender distribution of adult patients on highly active antiretroviral therapy (HAART) in Southern Africa: a systematic review. BMC Public Health 2007, 7:63.

31. WHO, UNAIDS, UNICEF: Global Update on HIV Treatment 2013: Results Impact and Opportunities. Geneva: WHO; 2013.

32. Van Dyk AC, van Dyk PJ: "To know or not to know": service-related barriers to voluntary HIV counseling and testing (VCT) in South Africa. Curationis 2003, 26:4-10.

33. Ramirez-Avila L, Nixon K, Noubary F, Giddy J, Losina E, Walensky RP, Bassett IV: Routine HIV testing in adolescents and young adults presenting to an outpatient clinic in Durban, South Africa. PLoS One 2012, 7:e45507.

34. Bassett IV, Giddy J, Wang B, Lu Z, Losina E, Freedberg KA, Walensky RP: Routine, voluntary HIV testing in Durban, South Africa: correlates of HIV infection. HIV Med 2008, 9:863-867.

35. Hendriksen ES, Hlubinka D, Chariyalertsak S, Chingono A, Gray G, Mbwambo J, Richter L, Kulich M, Coates TJ: Keep talking about it: HIV/AIDS-related communication and prior HIV testing in Tanzania, Zimbabwe, South Africa, and Thailand. AIDS Behav 2009, 13:1213-1221.

36. Phakathi Z, Van Rooyen H, Fritz K, Richter L: The influence of antiretroviral treatment on willingness to test: a qualitative study in rural KwaZulu-Natal, South Africa. African J AIDS Res 2011, 10:173-180.

37. McGrath N, Eaton JW, Bärnighausen TW, Tanser F, Newell M-L: Sexual behaviour in a rural high HIV prevalence South African community: time trends in the antiretroviral treatment era. AIDS 2013, 27:2461-2470.

38. Rosenberg NE, Westreich D, Bärnighausen T, Miller WC, Behets F, Maman S, Newell $M-L$, Pettifor A: Assessing the effect of HIV counselling and testing on HIV acquisition among South African youth. AIDS 2013, 27:2765-2773.

39. Baeten JM, Donnell D, Ndase P, Mugo NR, Campbell JD, Wangisi J, Tappero JW, Bukusi E a, Cohen CR, Katabira E, Ronald A, Tumwesigye E, Were E, Fife KH, Kiarie J, Farquhar C, John-Stewart G, Kakia A, Odoyo J, Mucunguzi A, Nakku-Joloba E, Twesigye R, Ngure K, Apaka C, Tamooh H, Gabona F, Mujugira A, Panteleeff D, Thomas KK, Kidoguchi L, et al: Antiretroviral prophylaxis for HIV prevention in heterosexual men and women. $N$ Engl J Med 2012, 367:399-410.
40. Padian NS, Mccoy SI, Karim SSA, Hasen N, Kim J, Bartos M, Katabira E, Bertozzi SM, Schwartländer B, Cohen MS, Gates M: Review HIV prevention transformed: the new prevention research agenda. Lancet 2011, 378:269-278.

41. Peck RB, Lim JM, van Rooyen H, Mukoma W, Chepuka L, Bansil P, Knight LC, Muturi N, Chirwa E, Lee AM, Wellhausen JD, Tulloch O, Taegtmeyer M: What should the ideal HIV self-test look like? A usability study of test prototypes in unsupervised HIV self-testing in Kenya, Malawi, and South Africa. AIDS Behav 2014, 18(Suppl 4):422-432.

42. Michielsen $K$, Chersich MF, Luchters S, De Koker P, Van Rossem R, Temmerman M: Effectiveness of HIV prevention for youth in sub-Saharan Africa: systematic review and meta-analysis of randomized and nonrandomized trials. AIDS 2010, 24:1193-1202.

43. Van Rooyen H, Barnabas RV, Baeten JM, Phakathi Z, Joseph P, Krows M, Hong T, Murnane PM, Hughes J, Celum C: High HIV testing uptake and linkage to care in a novel program of home-based HIV counseling and testing with facilitated referral in KwaZulu-Natal, South Africa. J Acquir Immune Defic Syndr 2013, 64:1-8.

doi:10.1186/1471-2458-14-1164

Cite this article as: Knight et al:: Characteristics of sexually experienced HIV testers aged 18 to 32 in rural South Africa: baseline results from a community-based trial, NIMH Project Accept (HPTN 043). BMC Public Health 2014 14:1164.

\section{Submit your next manuscript to BioMed Central and take full advantage of:}

- Convenient online submission

- Thorough peer review

- No space constraints or color figure charges

- Immediate publication on acceptance

- Inclusion in PubMed, CAS, Scopus and Google Scholar

- Research which is freely available for redistribution 